\title{
1. Title: Rotator cuff disease: opinion regarding surgical criteria and likely outcome
}

\section{Short title: Rotator cuff surgery: surgeon opinion}

Authors:

Alison Thorpe MPhil, BAppSc (physiotherapy), School of Physiotherapy and Exercise Science, Curtin University, Western Australia Mark Hurworth, FRACS, FA(Orth)A

Peter O'Sullivan, PhD, Grad Dip Manip Ther, School of Physiotherapy and Exercise Science, Curtin University, Western Australia Tim Mitchell, PhD, BAppSc (physiotherapy), School of Physiotherapy and Exercise Science, Curtin University, Western Australia Anne Smith, PhD, Masters (Biostatistics), School of Physiotherapy and Exercise Science, Curtin University, Western Australia

Alison Thorpe is a recipient of an Australian Postgraduate Award and a Curtin University Postgraduate Award to undertake a Doctor of Philosophy.

Abstract word count: 196 words

Main text word count: 3533 words

Tables included: 1

Supporting information:

Documents: Document S1

Document S2

Tables: $\quad$ Table S1

Corresponding author:

Alison Thorpe

Email: a.thorpe@curtin.edu.au

Postal address: Curtin University, School of Physiotherapy and Exercise Science, GPO Box U1987, Perth WA 6845

Telephone: (w) 0892669227 (m) 0412093715 


\section{Abstract}

Keywords: rotator cuff disease, surgeon opinion, surgical criteria

\section{Background}

Clinical guidelines for the management of rotator cuff disease are not clear. Surgeon surveys in the USA and UK lack agreement regarding surgical indications. Physical examination tests aid surgical decision-making but also lack robust evidence. Study aims were to evaluate: Western Australian (WA) Orthopaedic Surgeons' perceptions about surgical indications; utility of physical examination tests; findings at surgery predictive of outcome and surgeon opinion of a successful surgical outcome.

\section{Methods}

An anonymous rotator cuff survey, previously reported by the American Academy of Orthopaedic Surgeons, was emailed to all surgeons listed with the Australian Orthopaedic Association (AOA) in WA. Surgeons who treated patients for rotator cuff disease during the previous 12 months were invited to complete the rotator cuff survey and five additional questions were included to capture the above criteria of interest.

\section{Results}

Within a close community of surgeons based in Western Australia $(n=23)$ considerable heterogeneity exists in surgical decision-making criteria. A successful surgical outcome was considered to include reduced pain levels, restoration of movement and function and gains in muscle strength.

\section{Conclusions}

Research is required to inform robust clinical practice guidelines for rotator cuff surgery. Identification of prognostic factors for successful surgical outcome is imperative. 


\section{Introduction}

Shoulder pain due to rotator cuff disease $(R C D)$ is a frequently reported musculoskeletal complaint, with prevalence rates reported to be as high as $36 \%$ during adult life ${ }^{1}$. Surgery may be indicated after failure to respond to a conservative program that can include pharmacology, injection therapy, physiotherapy and exercise rehabilitation.

Surgical criteria for RCD are not clear, with clinical practice guidelines and surveys of surgeon opinion outlining a lack of consensus agreement between surgeons for the management of RCD 23 , 4. Evidence-based clinical guidelines, surgeon education, surgeon personal experience and individual patient factors, may inform surgical decision-making. The lack of robust clinical guidelines is a result of limited high quality evidence ${ }^{2,5}$.

Clinical examination tests, such as weakness or pain on muscle testing ${ }^{4}$ or clinical orthopaedic tests ${ }^{6-8}$ may assist with decision-making, however tests also lack robust evidence. A systematic review determined that most tests for RCD are inconclusive, with limited recommendation for their clinical use ${ }^{9}$.

Surgical views may be similar in the Australian orthopaedic community as the USA and UK, however surgeon opinion in Australia has not been reported. The aims of this study were to survey Western Australian (WA) orthopaedic surgeons' for opinions regarding (i) indications for rotator cuff surgery; (ii) physical examination tests most commonly utilised; (iii) findings at surgery that may be predictive of outcome; and (iv) what constitutes a successful surgical outcome.

\section{Methods}

A link to an anonymous rotator cuff survey, including questions previously reported by the American Academy of Orthopaedic Surgeons (AAOS) ${ }^{3}$ plus five additional questions, was emailed during December 2013 and January 2014 to all surgeons listed with the Australian Orthopaedic Association $(A O A)$ in WA. Surgeons who treated patients for RCD during the previous 12 months were invited to complete the survey, with a cover letter from an AOA surgeon and co-investigator, M.H. included with the survey to encourage surgeon participation, with consent implied by participation. An initial screening question determined that only surgeons having performed surgery for RCD during the previous 12 months were directed to proceed with the survey. Ethical approval 
was attained from the Human Research and Ethics Committee at Curtin University (approval number HR 178/2013).

The survey (Document S1) established surgeon characteristics (consultancy practice years, number of rotator cuff repairs $(\mathrm{RCR})$ performed during the past year, preferred method of repair for a $2 \mathrm{~cm}$ full thickness tear and estimated percentage annual failure rate (defined as patient dissatisfaction) for patients undergoing RCR in WA. Specific questions pertaining to RCD surgery included management of clinical case vignettes, opinion on clinical statements, number of steroid injections given safely in one year and factors facilitating greater patient involvement in surgical decisionmaking. The vignettes described four common clinical presentations, with a tear confirmed on magnetic resonance imaging, for which respondents were asked to select one of four management options; conservative treatment (with physiotherapy or cortisone injection) or surgery (with or without RCR). The clinical statements surveyed opinion about factors that might influence choice of management for patients with RCD and respondents were asked to rate the importance on a 5-point Likert scale (strongly disagree, disagree, indifferent, agree, and strongly agree). Responses for agree and strongly agree, and disagree and strongly disagree, were pooled to determine levels of clinical agreement or disagreement respectively. Clinical agreement was defined as $80 \%$ or higher consensus between surgeons answering similarly, as previously reported in the AAOS study ${ }^{3}$. Five additional questions were developed in consultation with WA surgeons: three questions regarding physical examination tests most commonly used for surgical decision-making; findings at the time of surgery predictive of outcome at 12 months; and what would be considered a successful outcome for repair of a $1 \mathrm{~cm}$ by $1 \mathrm{~cm}$ supraspinatus tear in the critical zone (Document S2).

\section{Results}

Twenty-six surgeons attempted the survey. Three respondents were excluded from further analysis (one indicated they had not performed shoulder surgery during the previous year and two only partly completed the survey), leaving a sample group of 23 respondents. While it is likely these respondents include all 24 surgeons listed on the AOA website as 'Shoulder and Elbow surgeons', suggesting a $100 \%$ response rate, the anonymous nature of the survey precludes this definitive conclusion. 


\section{Surgeon characteristics}

The mean number of rotator cuff surgeries undertaken was 80 cases per year (ranging from 12-200 cases with a median of 75 cases). The mean years of consultation practice was 8.5 years (ranging from 1 to 25 years with a median of 5 years). Annual estimated failure rate (defined as patient dissatisfaction) recorded a mean of $12 \%$ (ranging from $2-25 \%$ per year with a median of $10 \%$ ). The preferred surgical technique for repair of a $2 \mathrm{~cm}$ full thickness supraspinatus tear in the critical zone was mini-open $(n=17,74 \%)$ over arthroscopic $(n=4,16 \%)$ or open $(n=2,8 \%)$.

\section{Clinical Vignettes}

Clinical agreement (>80\%) was attained for only one vignette (vignette 2 ), with no consensus attained for management of the other three vignettes, with results summarised in Table 1.

\section{Clinical statements regarding surgery for $R C D$}

Only one statement achieved clinical agreement (>80\%); that the duration and frequency of rehabilitation should be discussed pre-operatively with the patient (Table S1).

\section{Steroid injections per year}

Most surgeons (70\%) believed that the maximum number of steroid injections safely administered in one year to patients with RCD was three injections. Other surgeons believed that one (4\%), two $(4 \%)$, four ( $9 \%)$ or five $(13 \%)$ could safely be given per year.

Factors for patients to be more involved in the decision-making process for surgery Most respondents (87\%) reported their patients are already sufficiently involved in the decisionmaking process, however the provision of more information (26\%), longer pre-operative appointments (13\%) and a higher patient education level (13\%) were also considered important factors.

\section{Physical examination tests used in decision-making for surgery}

Active range (96\%), Hawkins test $(87 \%)$, passive range (78\%), painful arc $(78 \%)$, isometric muscle tests $(57 \%)$, Neer sign (57\%), empty can test $(57 \%)$ and drop arm test $(52 \%)$ were identified as the tests most frequently utilised, with most surgeons using a cluster of four or more tests. 
Intra-operative findings considered predictive of 12-month outcome related primarily to the presence of tendon pathology (quality of tissue (65\%), degree of tendon retraction (39\%), ease of tendon reduction $(30 \%)$, size of tear $(35 \%)$ or irreparable tendon $(9 \%))$. Other factors observed intraoperatively included co-morbidities such as glenohumeral joint osteoarthritis (13\%), acromioclavicular joint pathology $(9 \%)$, presence of inflammation $(9 \%)$, long head of biceps pathology (4\%) or presence of fat atrophy $(4 \%))$.

\section{Surgeon classification of a successful outcome}

A successful surgical outcome was considered to relate to a cluster of three or more factors including minimal or no pain (74\%), restoration of range of active and passive range $(52 \%)$, return to functional activities (52\%), minimal strength deficit (39\%) and a healed tendon (13\%).

\section{Discussion}

Within a close community of surgeons based in Western Australia $(n=23)$ considerable heterogeneity exists in surgical decision-making criteria, consistent with previous studies from the USA and UK ${ }^{3,4}$. This study surveyed a younger surgeon sample (9 versus 18 years of consultancy practice in USA study), with a similar estimated failure rate (12\% versus $15 \%)^{3}$. The trend towards mini-open and arthroscopic procedures in this study is likely to reflect advances in surgical techniques utilised by more recently trained shoulder surgeons.

Clinical agreement was achieved for only vignette 2 in this study, whereas the previous USA study failed to achieve agreement for any vignette. For Vignette 1, less than half of surgeons surveyed (48\%) recommended surgery with RCR, despite weak evidence that early surgical repair for tear after traumatic injury is indicated ${ }^{2}$ and failure of a conservative physiotherapy program is considered to be an indication for surgery ${ }^{4}$. For Vignette 2, clinical agreement (83\%) was attained for surgery with RCR, in keeping with clinical guidelines recommending early surgical repair for full thickness tears after traumatic injury ${ }^{2}$. For Vignette 3 conservative management was recommended by $61 \%$, with physiotherapy (39\%) favoured over cortisone injection (22\%). Evidence for physiotherapy and corticosteroid injection is inconclusive ${ }^{2}$, however surveys of surgeon opinion suggest a minimum three months of physiotherapy prior to considering surgery ${ }^{3,4}$. Surgery with RCR was recommended by only $39 \%$ of surgeons, which may reflect their awareness of weak 
evidence for RCR in patients with chronic, symptomatic, full thickness tears ${ }^{2}$. For Vignette 4 approximately one third (35\%) recommended surgery with RCR despite the presence of poor tendon health on MRI, contrary to clinical guidelines that suggest the presence of fat atrophy on MRI correlates with less favourable outcome of surgery ${ }^{2}$.

The presence of pain is usually a reason why patients seek treatment, however a poor correlation between pain severity and rotator cuff tear severity has been reported ${ }^{10}$. Both vignette 2 and 3 report minimal pain levels, however twice as many surgeons recommended surgery for vignette 2 over vignette $3(83 \%$ versus $39 \%)$, suggesting the decision to operate is multifactorial. Traumatic onset, younger age, larger tendon tear and muscle weakness in vignette 2 may all influence surgical decision-making. Clinical guidelines report increasing age weakly correlates with less favourable outcomes after RCR surgery ${ }^{2,11}$. Younger age was a factor for consideration in vignette 1 and 2 with a young labourer reported to have a partial thickness tear in vignette 1 and a full thickness tear in vignette 2. Consensus agreement for surgery with RCR was attained on vignette 2 only (83\%), versus vignette 1 (48\%), indicating that the presence of a full thickness tear in vignette 2 more strongly influenced the decision for surgery than age. Age-related degenerative changes are considered part of the natural history of RCD, are frequently asymptomatic ${ }^{12,13}$ and full thickness tear prevalence in asymptomatic individuals over 60 is reported to range from $25 \%-50 \%$, increasing after 80 years ${ }^{12,14}$.

Clinical statements regarding surgery for RCD (Table S1) reached consensus (100\%) on only one factor (expected frequency and duration of post-operative rotator cuff rehabilitation should be discussed with patients pre-operatively) and showed a trend towards consensus (78\%) on another (surgeon should explain the options and let the patient decide whether to have surgery), which is aligned with previous results ${ }^{3}$. Consensus $(>80 \%)$ was not attained for the remaining seven statements: physiotherapy is useful for full thickness tears (61\% agreement); the use of a steroid injection is contraindicated in potential surgical candidates $(70 \%$ disagreement); patients should expect to have a normal shoulder after RCR (57\% disagreement); surgeon should decide for or against RCR (52\% disagreement); major reason for RCR is to prevent tear progression (52\% agreement) or osteoarthritis (61\% disagreement); and pros and cons of surgery should be 
discussed pre-operatively (48\% agreement, 39\% indifferent). The lack of consensus with clinical statements is despite evidence that exercise therapy has been associated with alleviation of symptoms in patients with full thickness tears ${ }^{15-17}$ and recommended for rotator cuff related symptoms, in the absence of full thickness tears ${ }^{2}$. Low patient expectations regarding the effectiveness of physiotherapy has been shown to be a strong predictor of the decision to undergo surgery ${ }^{18}$. Evidence for the use of corticosteroid injections in patients with rotator cuff tears is inconclusive ${ }^{2}$, with weak evidence supporting corticosteroid injection for $\mathrm{RCD}{ }^{19}$, however a recent systematic review suggests emerging evidence of significant long-term harm to tendon tissue with corticosteroid injection ${ }^{20}$. There was a lack of consensus for the role of RCR surgery to prevent tear progression or osteoarthritis. Tear progression has been reported in $50 \%$ of asymptomatic full thickness tears that go on to develop symptoms within 2-3 years ${ }^{12}$. The pathogenesis of rotator cuff arthropathy in conservatively managed tears has been reported to relate to the number of ruptured rotator cuff tendons ${ }^{21}$. However, poor correlation between pathological changes and functional status has been reported with superior migration of the humeral head and deterioration in tendon quality occurring without the loss of shoulder function ${ }^{21}$.

Multiple intra-operative factors were considered prognostic of outcome with tendon quality, tear size, location, ease of tendon repair and the presence of associated pathology considered most relevant. These findings are in keeping with clinical guidelines and surveys of surgical opinion undertaken in USA and UK that report that tear size and the presence of fat atrophy correlate with less favourable surgical outcome ${ }^{2,4}$. However these findings are at odds with a recent meta-analysis reporting that the structural integrity of the repaired tendon does not correlate with clinically important differences in patient function and pain relief after RCR ${ }^{22}$. Most surgeons agree that successful outcome includes reduced pain levels, restoration of movement and function and gains in muscle strength. Recent evidence suggests that shoulder pain is multifactorial and influenced by physical (movement behaviour, muscle control, mobility restriction $)^{23}$, psychological ${ }^{24}$, tissue sensitivity ${ }^{25,26}$ and lifestyle (such as general health, smoking, diet and physical activity) ${ }^{27}$ factors. Surgeons should be cognisant of multidimensional contributing factors, when deciding appropriate management for patients. 
Given the current lack of evidence supporting surgery over conservative management for RCD, a minimum period of three months physiotherapy-led education regarding load management and lifestyle factors and targeted exercise rehabilitation should be undertaken prior to surgery ${ }^{28-30}$. As patients who have low expectations regarding the effectiveness of physiotherapy are more likely to fail non-operative treatment ${ }^{18}$, surgeons should not only consider promoting physiotherapy management as a first choice, but also reinforce a positive expectation regarding the outcome of a conservative approach. Surgical criteria should include failure to respond to an extended course of physiotherapy-led exercise rehabilitation, as well as consideration of multifactorial contributors to outcomes such as mental health ${ }^{24}$. This will likely result in a reduction in unnecessary surgery, minimise the cost and risk of surgery from the patient's perspective and improve indications for patient selection for rotator cuff surgery.

Future studies that identify prognostic factors for conservative and surgical outcomes are imperative. The development of a model of care for shoulder pain, similar to the national strategy for osteoarthritis ${ }^{31}$, would facilitate an assessment process that includes general health, lifestyle and psychosocial screening alongside pathology specific considerations. Intervention studies targeting baseline physical, psychological or tissue sensitivity, such as physiotherapy-led exercise rehabilitation, cognitive functional approaches or targeted pharmacology may elucidate the influence of these factors on outcome.

There are a number of limitations noted in this study. Firstly, data collected was from the WA orthopaedic community resulting in a small sample size that may not be representative of surgical opinion throughout Australia, however findings are similar to those previously reported in the USA and UK. Secondly, only surgeons listed as members of the Australian Orthopaedic Association were invited to participate in the study and the sample group may not include all WA shoulder surgeons. Thirdly, the limited clinical information provided in the vignettes may lead to different interpretations between surgeons with regard to management choice. The rotator cuff survey was used as initially developed to allow comparison of responses between studies and changes in management views over the past decade.

\section{Conclusion}


Within a close community of surgeons based in Western Australia $(n=23)$ considerable heterogeneity exists in surgical decision-making criteria, that is consistent with a previous USA study, and highlight the lack of robust clinical guidelines to inform patient selection for rotator cuff surgery. Research is required to inform robust clinical practice guidelines for rotator cuff surgery. Identification of prognostic factors for successful surgical outcome is imperative.

\section{Acknowledgements}

Nil

\section{Disclosure Statement}

There are no conflicts of interest to disclose

\section{References}

1. Green S, Buchbinder R, Hetrick SE. Physiotherapy interventions for shoulder pain (Review). The Cochrane Collaboration. 2013; Available

2. Pedowitz RA, Yamaguchi K, Ahmad CS, Burks RT, Flatow EL, Green A, et al. American Academy of Orthopaedic Surgeons Clinical Practice Guideline on optimizing the management of rotator cuff problems. Journal of Bone and Joint Surgery Am. 2012; 94:163-167. Available

3. Dunn WR, Schackman BR, Walsh C, Lyman S, Jones EC, Warren RF, et al. Variations in orthopaedic surgeons' perceptions about the indications for rotator cuff surgery. Journal of Bone and Joint Surgery Am. 2005; 87(9):1978-1984. Available

4. Griffiths S, Yohannes A. Surgical referral criteria for degenerative rotator cuff tears: a Delphi questionnaire study. Musculoskeletal Care. 2014; 12:82-91. Available

5. Wright JG, Katz JN, Losina E. Clinical trials in orthopaedics research. Part I. Cultural and practical barriers to randomized trials in orthopaedics. Journal of Bone and Joint Surgery Am [Research Support, N.I.H., Extramural

Research Support, Non-U.S. Gov't]. 2011; 93(5):e15. DOI:10.2106/JBJS.J.00229.

6. Papadonikolakis A, McKenna M, Warme W, Martin BI, Matsen FA. Published evidence relevant to the diagnosis of impingement syndrome of the shoulder. Journal of Bone and Joint Surgery Am. 2011; 93:1827-1832. DOI:10.2106/JBJS.J.01748.

7. Neer CS. Impingement lesions. Clinical Orthopaedics and Related Research. 1983; 173:70-77. Available

8. Hawkins RJ, Kennedy JC. Impingement syndrome in athletes. American Journal of Sports Medicine. 1980; 8:151-158. Available

9. Hughes PC, Taylor NF, Green RA. Most clinical tests cannot accurately diagnose rotator cuff pathology: a systematic review. Australian Journal of Physiotherapy. 2008; 54(3):159-170. DOI:10.1016/s0004-9514(08)70022-9.

10. Dunn WR, Kuhn JE, Sanders R, An Q, Baumgarten KM, Bishop JY, et al. Symptoms of pain do not correlate with rotator cuff tear severity: a cross-sectional study of 393 patients with a symptomatic atraumatic full-thickness rotator cuff tear. J Bone Joint Surg Am. 2014; 96(10):793-800. DOI:10.2106/JBJS.L.01304.

11. Seida J, Schouten J, Mousavi S, Tjosvold L, Vandermeer B, Milne A, et al. Comparative effectiveness of nonoperative and operative treatment for rotator cuff tears. Comparative Effectiveness Review No. 22. (Prepared by the University of Alberta Evidence-based Practice Center under Contract No. 
290-02-0023.) AHRQ Publication No. 10-EHC050. Rockville, MD: Agency for Healthcare Research and Quality. 2010; Available from: http://www.effectivehealthcare.ahrq.gov/reports/final.cfm.

12. Tashjian RZ. Epidemiology, natural history, and indications for treatment of rotator cuff tears. Clinics in Sports Medicine [Research Support, Non-U.S. Gov't

Research Support, U.S. Gov't, Non-P.H.S.

Review]. 2012; 31(4):589-604. DOI:10.1016/j.csm.2012.07.001.

13. Yamaguchi K, Tetro A, Blam O, Evanoff B, Teefey S, Middleton W. Natural history of asymptomatic tears:a longitudinal analysis of asymptomatic tears detected sonographically. Journal of Shoulder and Elbow Surgery. 2001; 10:199-203. Available

14. Sher JS, Uribe JW, Posada A, Murphy BJ, Zlatkin MB. Abnormal findings on magnetic resonance imaging of asymptomatic shoulders. Journal of Bone and Joint Surgery. 1995; 77:10-15. Available

15. Ainsworth R, Lewis JS. Exercise therapy for the conservative management of full thickness tears of the rotator cuff: a systematic review. $\mathrm{Br} J$ Sports Med. 2007; 41(4):200-10. DOI:10.1136/bjsm.2006.032524.

16. Magarey ME. A physiotherapy perspective on management of degenerative rotator cuff tendinopathy. Techniques in Shoulder and Elbow Surgery. 2012; 13(3):111-122. Available

17. Kuhn JE, Dunn WR, Sanders R, An Q, Baumgarten KM, Bishop JY, et al. Effectiveness of physical therapy in treating atraumatic full-thickness rotator cuff tears: a multicenter prospective cohort study. J Shoulder Elbow Surg. 2013; 22(10):1371-9. DOI:10.1016/j.jse.2013.01.026.

18. Dunn WR, Kuhn JE, Sanders R, An Q, Baumgarten KM, Bishop JY, et al. 2013 Neer Award: predictors of failure of nonoperative treatment of chronic, symptomatic, full-thickness rotator cuff tears. J Shoulder Elbow Surg. 2016; 25(8):1303-11. DOI:10.1016/j.jse.2016.04.030.

19. Buchbinder R, Green S, Youd JM. Corticosteroid injections for shoulder pain. Cochrane Database Systematic Review. 2003; 1:CD004016. Available

20. Dean BJ, Lostis E, Oakley T, Rombach I, Morrey ME, Carr AJ. The risks and benefits of glucocorticoid treatment for tendinopathy: a systematic review of the effects of local glucocorticoid on tendon. Semin Arthritis Rheum. 2014; 43(4):570-6. DOI:10.1016/j.semarthrit.2013.08.006.

21. Zingg PO, Jost B, Sukthankar A, Buhler M, Pfirrmann CW, Gerber C. Clinical and structural outcomes of nonoperative management of massive rotator cuff tears. J Bone Joint Surg Am. 2007; 89(9):1928-34. DOI:10.2106/JBJS.F.01073.

22. Russell RD, Knight JR, Mulligan E, Khazzam MS. Structural integrity after rotator cuff repair does not correlate with patient function and pain: a meta-analysis. J Bone Joint Surg Am. 2014; 96(4):265-71. DOI:10.2106/JBJS.M.00265.

23. Lewis J, McCreesh K, Roy JS, Ginn K. Rotator Cuff Tendinopathy: Navigating the DiagnosisManagement Conundrum. J Orthop Sports Phys Ther. 2015:1-43. DOI:10.2519/jospt.2015.5941.

24. Chester R, Jerosch-Herold C, Lewis J, Shepstone L. Psychological factors are associated with the outcome of physiotherapy for people with shoulder pain: a multicentre longitudinal cohort study. British Journal of Sports Medicine. 2016:bjsports-2016-096084. DOI:10.1136/bjsports-2016-096084.

25. Plinsinga ML, Brink MS, Vicenzino B, van Wilgen CP. Evidence of Nervous System Sensitization in Commonly Presenting and Persistent Painful Tendinopathies: A Systematic Review. J Orthop Sports Phys Ther. 2015; 45(11):864-75. DOI:10.2519/jospt.2015.5895.

26. Sanchis MN, Lluch E, Nijs J, Struyf F, Kangasperko M. The role of central sensitization in shoulder pain: A systematic literature review. Semin Arthritis Rheum. 2015; 44(6):710-6. DOI:10.1016/j.semarthrit.2014.11.002.

27. Bishop JY, Santiago-Torres JE, Rimmke N, Flanigan DC. Smoking Predisposes to Rotator Cuff Pathology and Shoulder Dysfunction: A Systematic Review. Arthroscopy. 2015; 31(8):1598-605. DOI:10.1016/j.arthro.2015.01.026.

28. Saltychev M, Aarimaa V, Virolainen P, Laimi K. Conservative treatment or surgery for shoulder impingement: systematic review and meta-analysis. Disabil Rehabil. 2015; 37(1):1-8. DOI:10.3109/09638288.2014.907364.

29. Kukkonen J, Joukainen A, Lehtinen J, Mattila KT, Tuominen EKJ, Kauko T, et al. Treatment of non-trauamtic rotator cuff tears. A randomised controlled trial with one-year clinical results. The Bone and Joint Journal. 2014; 96-B(1):75-81. DOI:10.1302/0301-620X.96B1. 
30. Kukkonen J, Joukainen A, Lehtinen J, Mattila KT, Tuominen EK, Kauko T, et al. Treatment of nontraumatic rotator cuff Tears: a randomized controlled trial with two years of clinical and imaging follow-up. Journal of Bone and Joint Surgery. 2015; 97-A(21):1729-1737. DOI:10.2106/JBJS.N.01051.

31. Arthritis Australia. Time to Move: Osteoarthritis. A national strategy to reduce a costly burden, 2014

\section{Figure legends}

Nil

\section{Supporting information}

Document S1: Rotator Cuff Survey part 1

Document S2: Rotator Cuff Survey part 2

Table S1:

Clinical agreement regarding factors affecting surgical decision-making. 